\title{
Two-flavour lattice QCD correlation functions in the deconfinement transition region
}

\author{
Bastian B. Brandt \\ Institut für theoretische Physik, Universität Regensburg, D-93040 Regensburg \\ E-mail: brandtekph.uni-mainz.de
}

\section{Anthony Francis*}

Institut für Kernphysik, Johannes Gutenberg-Universität Mainz, D-55099 Mainz

Helmholtz Institut Mainz, Johannes Gutenberg-Universität Mainz, D-55099 Mainz.

E-mail: francis@kph.uni-mainz.de

\section{Harvey B. Meyer and Hartmut Wittig}

PRISMA Cluster of Excellence, Johannes Gutenberg-Universität Mainz, D-55099 Mainz

Institut für Kernphysik, Johannes Gutenberg-Universität Mainz, D-55099 Mainz

Helmholtz Institut Mainz, Johannes Gutenberg-Universität Mainz, D-55099 Mainz

E-mail: meyerh@kph.uni-mainz.de and wittig@kph.uni-mainz.de

\begin{abstract}
We report on a lattice QCD calculation with two dynamical flavors of the isovector vector correlator in the high-temperature phase. We analyze the correlator in terms of the associated spectral function by performing a fit for the difference of the thermal and vacuum spectral functions, using also an exact sum rule that constrains this difference. Additonally we carry out a direct fit for the thermal spectral function, and obtain good agreement between the two analyses for frequencies below the two-pion threshold. Under the assumption that the spectral function is smooth in that region, we give an estimate of the electrical conductivity.
\end{abstract}

Xth Quark Confinement and the Hadron Spectrum,

October 8-12, 2012

TUM Campus Garching, Munich, Germany

\footnotetext{
* Speaker.
} 


\section{Introduction}

The properties of strongly interacting matter under extreme conditions are the subject of intensive experimental and theoretical investigation. A comprehensive picture of a state of matter requires not only the knowledge of equilibrium properties such as the equation of state and static susceptibilities, but also an understanding of its transport properties.

Here we report on recent progress in determining the Euclidean isovector vector correlation function using dynamical light quarks in the high temperature phase of QCD. This enables the first analysis of its underlying spectral function with unquenched quarks. We are able to determine the gross features of the thermal spectral function by analyzing directly the difference of the thermal and vacuum correlators. This difference can be further constrained using a recently derived sum rule [1]. In addition we employ an approach followed recently in a quenched study to determine the spectral function directly from the thermal correlator based on an appropriate Ansatz [2 - 4].

Note, this proceedings article represents a shortened version of [5] and we refer the reader to this publication for a more in depth discussion.

\section{Basic definitions and expectations}

Our primary observables are the Euclidean vector current correlators and their spectral representation:

$$
G_{\mu v}(\tau, T)=\int d^{3} x\left\langle J_{\mu}(\tau, \vec{x}) J_{v}(0)^{\dagger}\right\rangle=\int_{0}^{\infty} \frac{d \omega}{2 \pi} \rho_{\mu v}(\omega, T) \frac{\cosh [\omega(\beta / 2-\tau)]}{\sinh (\omega \beta / 2)}
$$

with $J_{\mu}(x) \equiv \frac{1}{\sqrt{2}}\left(\bar{u}(x) \gamma_{\mu} u(x)-\bar{d}(x) \gamma_{\mu} d(x)\right)$ the isospin current. For a given function $\rho(\omega, T)$, the reconstructed correlator is defined as

$$
G^{\mathrm{rec}}\left(\tau, T ; T^{\prime}\right) \equiv \int_{0}^{\infty} \frac{d \omega}{2 \pi} \rho\left(\omega, T^{\prime}\right) \frac{\cosh \left[\omega\left(\frac{\beta}{2}-\tau\right)\right]}{\sinh (\omega \beta / 2)} .
$$

It can be interpreted as the Euclidean correlator that would be realized at temperature $T$ if the spectral function was unchanged between temperature $T$ and $T^{\prime}$. For $T^{\prime}=0$ it can be directly obtained from the zero-temperature Euclidean correlator via [6]

$$
G^{\mathrm{rec}}(\tau, T) \equiv G^{\mathrm{rec}}(\tau, T ; 0)=\sum_{m \in \mathbb{Z}} G(|\tau+m \beta|, T=0) .
$$

In the thermodynamic limit, the subtracted vector spectral function obeys a sum rule (see [1] sec. $3.2)$,

$$
\int_{-\infty}^{\infty} \frac{d \omega}{\omega} \Delta \rho(\omega, T)=0, \quad \Delta \rho(\omega, T) \equiv \rho_{i i}(\omega, T)-\rho_{i i}(\omega, 0) .
$$

The electrical conductivity of $\mathrm{QCD}$, connected to the isospin diffusion constant $D$, is given by a Kubo formula in terms of the low-frequency behavior of the spectral function, (where $C_{e m}=$ $\left.\sum_{f=u, d} Q_{f}^{2}=5 / 9\right)$

$$
\sigma=C_{e m} D \chi_{s}=\frac{C_{e m}}{6} \lim _{\omega \rightarrow 0} \frac{\rho_{i i}(\omega, T)}{\omega}
$$




\begin{tabular}{|c|c|c|c|c|c|}
\hline & $64^{3} \times 128$ & Ref. & & $64^{3} \times 128$ & $64^{3} \times 16$ \\
\hline $6 / g_{0}^{2}$ & 5.50 & & $T[\mathrm{MeV}]$ & & $253(4)$ \\
\hline$\kappa$ & 0.13671 & & $\chi_{s} / T^{2}$ & & $0.871(1)$ \\
\hline$c_{\mathrm{sw}}$ & 1.7515 & & $A_{1} / T^{3}$ & $4.42(31)$ & \\
\hline$Z_{V}$ & $0.768(5)$ & [7] & $m_{1} / T$ & $3.33(5)$ & \\
\hline$a[\mathrm{fm}]$ & $0.0486(4)(5)$ & [8] & $\kappa_{0}$ & $1.244(5)$ & \\
\hline$m_{\pi}[\mathrm{MeV}]$ & 270 & [8] & $\Omega / T$ & $5.98(11)$ & \\
\hline
\end{tabular}

Table 1: The left part of the table shows the common quantities characterizing the zero-temperature and finite-temperature ensembles. In the right part, the fit parameters for the vacuum correlator in units of $T=253 \mathrm{MeV}$ and the value of the (isospin) quark number susceptibility $\chi_{s} / T^{2}$ are given. For more details on the generation of the $N_{\tau}=128$ ensemble, see [8]. The number of configurations generated with $N_{\tau}=16$ is 317 .

\section{Lattice QCD data}

All our numerical results were computed on dynamical gauge configurations with two light, mass-degenerate $\mathscr{O}(a)$-improved Wilson quark flavors. We calculated correlation functions using the same discretization and masses as in the sea sector in two different ensembles. The first corresponds to virtually zero-temperature on a $64^{3} \times 128$ lattice (labeled O7 in [8]) with a lattice spacing of $a=0.0486(4)(5) \mathrm{fm} \mathrm{[8]} \mathrm{and} \mathrm{a} \mathrm{pion} \mathrm{mass} \mathrm{of} m_{\pi}=270 \mathrm{MeV}$, so that $m_{\pi} L=4.2$. Secondly we generated an ensemble on a lattice of size $64^{3} \times 16$ with all bare parameters identical to the zero-temperature ensemble. In this way it is straightforward to compare the correlation functions respectively in the confined and deconfined phases of QCD. Choosing $N_{\tau}=16$ yields a temperature of $T \simeq 250 \mathrm{MeV}$. Based on preliminary results on the pseudo-critical temperature $T_{c}$ of the crossover from the hadronic to the high-temperature phase [9], the temperature can also be expressed as $T / T_{c} \approx 1.2$.

The vacuum correlator serves as a reference in this work. To fix the parameters of the lightest vector state in the finite volume of the simulation, we fitted the vacuum correlation function to an Ansatz of the form ${ }^{1}$

$$
G_{i i}(\tau, 0)=A_{1} e^{-m_{1} \tau}+\frac{3}{4 \pi^{2}} \kappa_{0} \exp (-\Omega \tau)\left(\Omega^{2} / \tau+2 \Omega / \tau^{2}+2 / \tau^{3}\right) .
$$

In addition we estimate the thermal (isovector) quark number susceptibility $\chi_{s} / T^{2}$ from the time-time component of the vector correlation function. The parameters used in our lattice setup, the ' $\rho$-meson' parameters and the value of the static susceptibility are summarized in Tab. 1.

\subsection{Thermal and vacuum correlators}

In Fig. 1(left) we show the correlator $G_{i i}(\tau, T)$ computed at $T \simeq 250 \mathrm{MeV}$ together with the corresponding free 'continuum' and free 'lattice discretized' correlation functions. In addition we show the reconstructed correlator $G_{i i}^{r e c}(\tau)$ as obtained from Eq. 2.3. The reconstructed correlator lies somewhat lower than the thermal correlator. The insert in Fig. 1 displays the ratio $G_{i i}(\tau) / G_{i i}^{r e c}(\tau)$ in order to make their relative $\tau$ dependence visible. For small Euclidean times

\footnotetext{
${ }^{1}$ For details please see [5]
} 

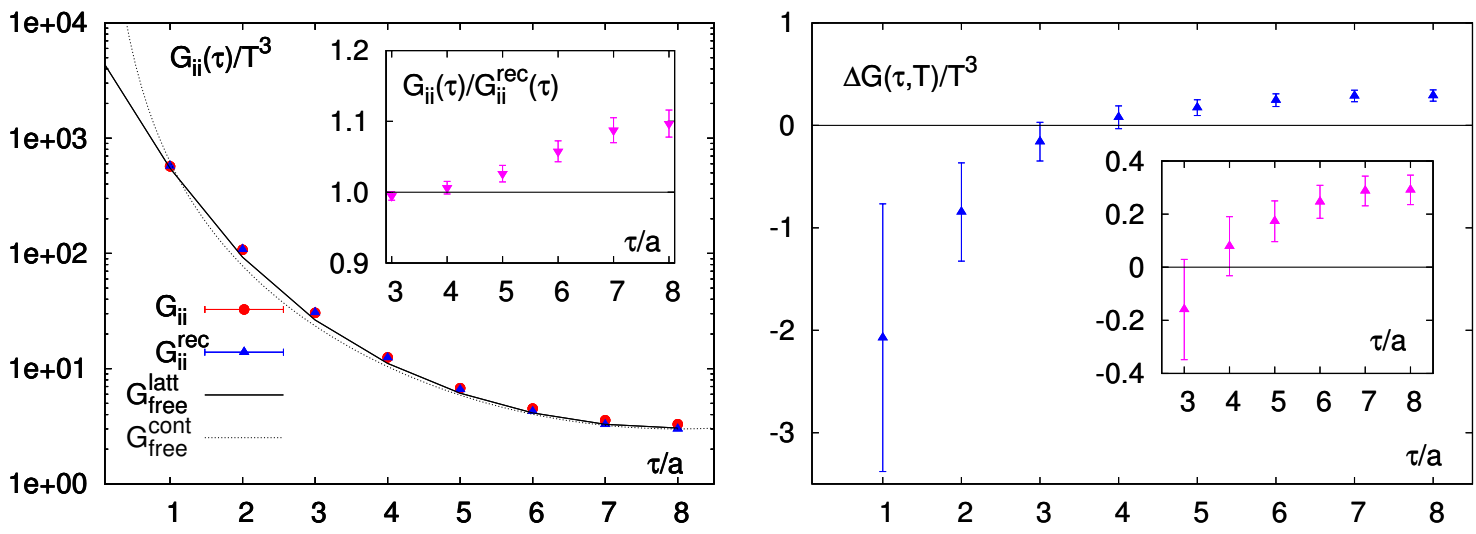

Figure 1: Left: Thermal $G_{i i}(\tau) / T^{3}$ and reconstructed $G_{i i}^{\text {rec }}(\tau) / T^{3}$ vector correlators over Euclidean time separation $\tau$ compared to the free (continuum and lattice) cases. The reconstructed correlator was computed by applying Eq. 2.3 to the data obtained from a lattice sized $N_{\sigma}=64$ and $N_{\tau}=128$. The insertion shows the ratio $G_{i i}(\tau) / G_{i i}^{\text {rec }}(\tau)$. Right: Difference $\Delta G(\tau, T) / T^{3}$ of the thermal vector correlator and the corresponding reconstructed correlator as a function of Euclidean time $\tau$. The insertion shows $\Delta G(\tau, T) / T^{3}$ in the region $\tau / a \geq 3$.

$\tau<\beta / 4$ this ratio is unity, above it increases monotonically until it levels off around the midpoint at about $10 \%$ above unity. A thermal modification of the spectral function has thus taken place (recall that the spectral function underlying $G_{i i}^{r e c}(\tau)$ contains the bound states of the confined theory). In Fig. 1(right) we show the difference

$$
\Delta G(\tau, T) \equiv G_{i i}(\tau, T)-G_{i i}^{r e c}(\tau, T)=\int_{0}^{\infty} \frac{d \omega}{2 \pi} \Delta \rho(\omega, T) \frac{\cosh [\omega(\beta / 2-\tau)]}{\sinh (\omega \beta / 2)}
$$

of the thermal and the reconstructed correlators. Given that $\rho_{i i}(\omega, T)$ and $\rho_{i i}(\omega, T=0)$ have the same $\sim \omega^{2}$ behavior, this means we are subtracting non-perturbatively the ultraviolet tail of the spectral function. Using this difference we are therefore able to probe the change in the vector spectral function from the confined to the deconfined phase for frequencies $\omega \lesssim \mathrm{O}(T)$.

For small times, the difference (3.2) turns out to be negative, while it is positive for $\tau \geq \beta / 4$. Note the errors decrease with increasing Euclidean time throughout the available range. We show a more detailed view of the region $\tau / a \geq 3$ in the insert of Fig. 1(right). Here the difference still exhibits a mild increase and levels off near the midpoint. The value it reaches at the midpoint is $\Delta G(\tau=\beta / 2, T) / T^{3}=0.291(55)$.

\section{Analysis of lattice correlators in terms of spectral functions}

\subsection{Fit to the thermal part of the vector correlator}

We proceed to investigate the behavior of the thermal part of the spectral function $\Delta \rho$ by fitting the difference of the thermal and the reconstructed correlator, see Eq. (3.2). As described in the previous subsection, the fact that the data (displayed in Fig. 1(right)) is positive at long distances and negative at short distances suggests that the thermal spectral weight exceeds the vacuum spectral weight at low frequencies and falls short of it at higher frequencies. 

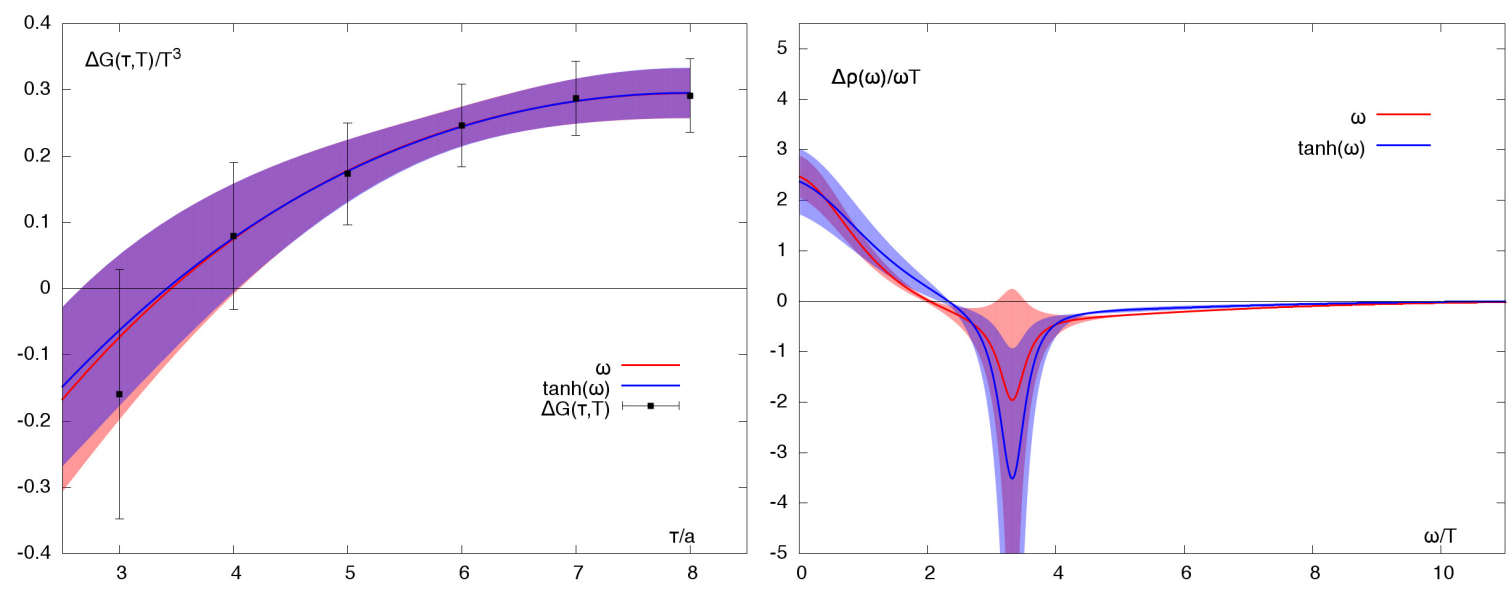

Figure 2: Left: Fits to $\Delta G(\tau, T) / T^{3} \equiv\left[G_{i i}(\tau)-G_{i i}^{r e c}(\tau)\right] / T^{3}$. The blue and red results differ by the form of transport peak in the Ansatz. The error bands are computed from the covariance matrix of the fit. Right: The resulting spectral functions for both Ansätze.

We thus parametrize $\Delta \rho$ using the following Ansatz for $\omega \geq 0$ :

$$
\begin{array}{rlrl}
\Delta \rho(\omega, T) & =\rho_{T}(\omega, T)-\rho_{B}(\omega, T)+\Delta \rho_{F}(\omega, T), \\
\rho_{B}(\omega, T) & =\frac{2 c_{B} g_{B} \tanh (\omega / T)^{3}}{4\left(\omega-m_{B}\right)^{2}+g_{B}^{2}}, & & \\
\rho_{T, 1}(\omega, T) & =\frac{4 c \omega}{(\omega / g)^{2}+1}, & \rho_{T, 2}(\omega, T)=\frac{4 c T \tanh (\omega / T)}{(\omega / g)^{2}+1}, \\
\Delta \rho_{F}(\omega, T) & =\rho_{F}(\omega, T)-\rho_{F}(\omega, 0), & \rho_{F}(\omega, T)=\frac{3}{2 \pi} \kappa \omega^{2} \tanh \left(\frac{\omega}{4 T}\right) .
\end{array}
$$

The bound state (B) and the transport peak (T) are represented by Breit-Wigner forms. Even such a simple Ansatz requires three parameters $\left(c_{B}, g_{B}, m_{B}\right)$ to determine the bound state peak, two parameters $(c, g)$ for the transport peak and one $(\kappa)$ for the 'perturbative' contribution $(\mathrm{F})$. We will therefore fix some of them using the vacuum correlator. In the following we set $m_{B}$ equal to $m_{1}$, given in Tab. 1, which we obtained from the exponential fit to the vacuum correlator. Note that the area under the bound state peak $\int d w \rho_{B} / \omega$ does not depend on the width $g_{B}$ in the limit where it is small. We therefore perform fits for three fixed values of this parameter, and check the sensitivity of the result. We choose the values $g_{B} / T=0.1,0.5$ and 1.0.

The tail $\sim T / \omega$ of the Ansatz $\rho_{T, 1}$ violates the OPE prediction that $\Delta \rho \sim(T / \omega)^{2}$ at large frequencies. It has been argued in [10] that this might lead to an overestimate of the transport contribution. To avoid this problem we introduce the Ansatz 2, where $\omega \rightarrow T \tanh (\omega / T)$. This Ansatz possesses the correct asymptotic behavior, as well as the expected linear behavior in $\omega$ at small frequencies. Finally, to complete the parametrization of $\Delta \rho(\omega)$, we include a weak-coupling term describing the subtraction of the large frequency parts of the thermal and vacuum spectral functions. This contribution $\rho_{F}(\omega, \kappa) \rightarrow 0$ vanishes exponentially as the frequency increases.

In the next step we fit the combined Ansätze of $\Delta \rho\left(\omega, c_{B}, g_{B}, m_{B}, c, g, \kappa\right)$ to the data, while at the same time satisfying the sum rule of Eq. 2.4 to an accuracy of $10^{-8}$. We limit ourselves to 

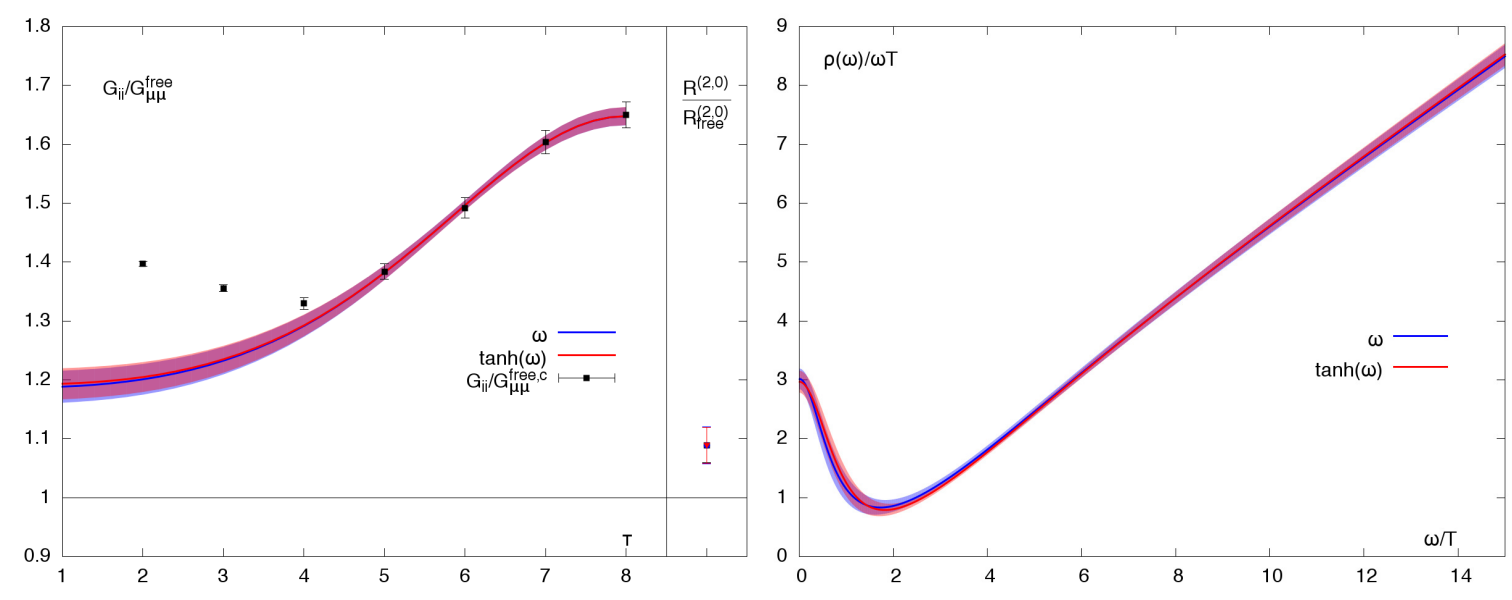

Figure 3: Left panel: fit results for $G_{i i}(\tau) / G_{\mu \mu}^{\text {free }}(\tau)$ using both Ansätze for the transport peak. The resulting ratio of thermal moments $R^{(2,0)} / R^{(2,0)}$ free are displayed on the right side of the plot. Right panel: The corresponding spectral functions normalized by $\omega T$.

fitting the region $5 \leq \tau / a \leq 8$ only, in order to minimize the influence of cut-off effects. With $m_{B}$ determined by the vacuum correlator, we set $g_{B}$ successively to the three different values mentioned above and fixed $\kappa$ around unity, and fitted the parameters $c, g$ and $c_{B}$. The errors and error bands shown in the following have been computed using the covariance matrix of the corresponding fit for fixed values of $g_{B}$ and $\kappa$.

The resulting correlators and spectral functions are displayed in Fig. 2 for $g_{B} / T=0.50$ and $\kappa=1.10$. In the left panel of Fig. 2 the data $\Delta G(\tau, T)$ is compared to the fits using $\rho_{T, 1}(\omega)$ and $\rho_{T, 2}(\omega)$ as transport contribution. We achieve a quasi-perfect description of the data for $\tau / a \geq 4$. The right panel shows that both Ansätze exhibit a substantial spectral weight around the origin and a negative contribution from the region of the $\rho$ mass.

\subsection{Weak-coupling inspired fit to the thermal vector}

In contrast to the previous section, here we study directly the thermal vector correlator and its ratio of thermal moments $R^{(2,0)}$, computed along the lines of [2]. We perform a fit inspired by the weak coupling form of the thermal spectral function,

$$
\rho(\omega, T)=\rho_{T}(\omega, T)+\rho_{F}(\omega, T)
$$

where the form of the two contributions is defined in Eq. (4.3) and (4.4). At a given temperature this Ansatz is characterized by three parameters $(c, g, \kappa)$. We fit the full Ansatz $\rho(\omega, c, g, \kappa)$ to the thermal correlator $G_{i i}(\tau)$, while at the same time demanding that $R^{(2,0)}$ be reproduced. In this analysis the three parameters $c, g$ and $\kappa$ are fitted, and the fit range is $5 \leq \tau / a \leq 8$ as before.

The resulting correlators and spectral functions are shown in Fig. 3. The ratio $G_{i i}(\tau) / G_{\mu \mu}^{\mathrm{free}}(\tau)$ in the left panel of Fig. 3 is well described by both versions $\rho_{T, 1}$ and $\rho_{T, 2}$ of the transport contribution for $\tau / a \geq 5$, while also the ratio of thermal moments (given on the far right of the plot) is reproduced. For $\tau / a<5$ our Ansatz fails to reproduce these points, which we suspect is partly due to cutoff effects. 


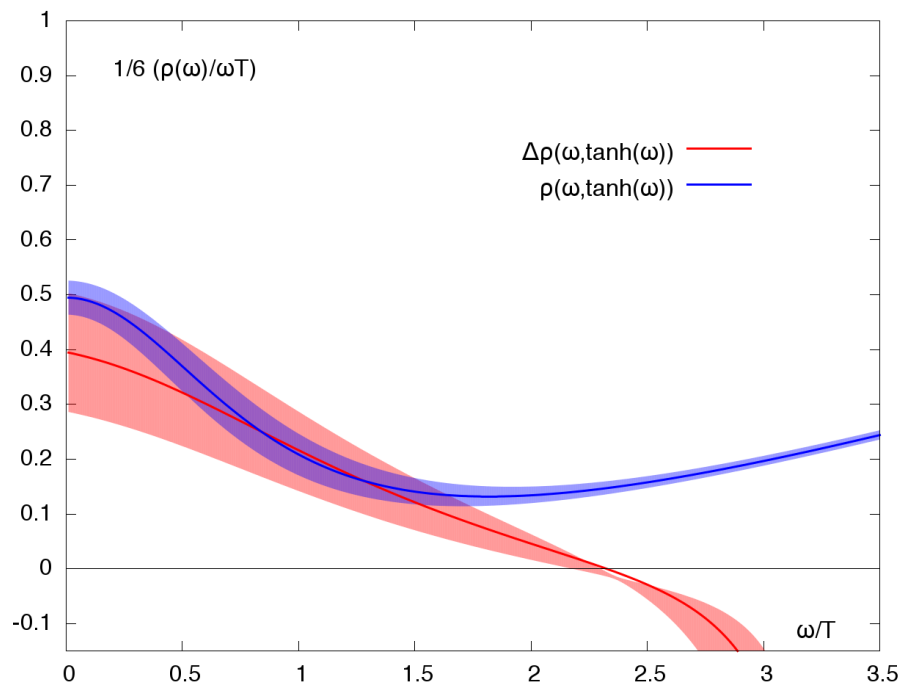

Figure 4: Comparison of the spectral functions obtained from analyzing (a) $\Delta G(\tau, T)$ and (b) $G_{i i}(\tau, T)$ using in both cases $\rho_{T, 2}(\omega) \sim \tanh (\omega / T)$ in the low frequency region. All curves have been multiplied by $a$ factor $1 / 6$ and divided by $\omega T$, entailing that the intercept at $\omega=0$ yields an estimate of $\sigma / C_{e m} T$.

On the right hand side of Fig. 3 we show the resulting spectral functions divided by $\omega T$. Clearly both Ansätze give very similar results that lie within errors of each other. The thermal correlator is even less sensitive to the asymptotic behavior of the transport contribution in the Ansatz than in the difference of correlators studied in Sec. 4.1.

\section{Discussion}

We now compare the results of the fits to $\Delta G(\tau, T)$ and $G_{i i}(\tau, T)$. Since the vacuum spectral function vanishes below $2 m_{\pi} \approx 540 \mathrm{MeV}$ (in infinite volume), $\rho_{i i}(\omega, T)$ and $\Delta \rho(\omega, T)$ should be equal for $\omega<2 m_{\pi} \approx 2.1 T$. We thus plot the spectral functions obtained from the two fits in this frequency region, see Fig. 4. Here we restrict ourselves to showing these results based on $\rho_{T, 2}(\omega, T)$, as their theoretical foundation is more sound than those with $\rho_{T, 1}(\omega, T)$. All curves are multiplied by a factor $1 / 6$, which means that the intercept at $\omega=0$ yields an estimate of $\sigma / C_{e m} T$ with $\sigma$ the electrical conductivity of the quark gluon plasma.

The results obtained by fitting $G_{i i}(\tau, T)$ agree very well with the central values obtained by fitting $\Delta G(\tau, T)$, whereby the fit to $\Delta G(\tau, T)$ using the transport Ansatz $\rho_{T, 2}(\omega)$ yields a slightly lower intercept. If we assume the spectral function to be as smooth around the origin as Fig. 4 suggests, we obtain the following estimate for the electrical conductivity of the quark gluon plasma at $T \simeq 250 \mathrm{MeV}$,

$$
\frac{\sigma}{C_{e m} T}=0.40(12)
$$

where $C_{e m}=\sum_{f=u, d} Q_{f}^{2}$. Although obtained under a strong assumption, it is interesting to compare (5.1) to other lattice results obtained under similar assumptions. The following comparison is made with quenched results, since to our knowledge there are no previous dynamical QCD studies. 
Quenched calculations using staggered fermions and different methods for analyzing the spectral function obtained $\sigma / T=7 C_{e m}[11]$ and $\sigma / T=0.4(1) C_{e m}$ [12] in the temperature region $1.5 \leq$ $T / T_{c} \leq 3$.0. Also, a recent quenched study using Wilson-Clover fermions in the continuum limit obtained $0.33 C_{e m} \leq \sigma / T \leq 1 C_{e m}$ at $T \simeq 1.45 T_{c}$ [2] and $T \simeq 1.1 T_{c}$ [4], with similar non-continuum results up to temperatures $T \simeq 2.98 T_{c}$ [3]. Our results using dynamical Wilson-Clover fermions at $N_{\tau}=16$ are thus completely compatible with the recent quenched results.

\section{Acknowledgments}

Computations were carried out on the JUGENE computer of the Gauss Centre for Supercomputing located at Forschungszentrum Jülich, Germany, within NIC project HMZ21, and the dedicated QCD platform "Wilson" at the Institute for Nuclear Physics, University of Mainz. This work was supported by the Center for Computational Sciences in Mainz and by the DFG grant ME 3622/2-1 Static and dynamic properties of QCD at finite temperature.

\section{References}

[1] D. Bernecker and H. B. Meyer, Vector Correlators in Lattice QCD: Methods and applications, Eur.Phys.J. A47 (2011) 148, [arXiv:1107.4388]. 16 pages, 9 figure files.

[2] H.-T. Ding, A. Francis, O. Kaczmarek, F. Karsch, E. Laermann, et. al., Thermal dilepton rate and electrical conductivity: An analysis of vector current correlation functions in quenched lattice QCD, Phys.Rev. D83 (2011) 034504, [arXiv: 1012 .4963].

[3] A. Francis and O. Kaczmarek, On the temperature dependence of the electrical conductivity in hot quenched lattice QCD, Prog.Part.Nucl.Phys. 67 (2012) 212-217, [arXiv:1112 . 4802].

[4] H. T. Ding et. al., Thermal dilepton rates from lattice QCD, This proceedings (2013).

[5] B. B. Brandt, A. Francis, H. B. Meyer, and H. Wittig, Thermal Correlators in the $\rho$ channel of two-flavor QCD, arXiv:1212.4200.

[6] H. B. Meyer, The Bulk Channel in Thermal Gauge Theories, JHEP 04 (2010) 099, [arXiv:1002.3343].

[7] M. Della Morte, R. Hoffmann, F. Knechtli, R. Sommer, and U. Wolff, Non-perturbative renormalization of the axial current with dynamical Wilson fermions, JHEP 0507 (2005) 007, [hep-lat/0505026].

[8] P. Fritzsch, F. Knechtli, B. Leder, M. Marinkovic, S. Schaefer, et. al., The strange quark mass and Lambda parameter of two flavor QCD, arXiv: 1205.5380.

[9] B. B. Brandt, A. Francis, H. B. Meyer, H. Wittig, and O. Philipsen, QCD thermodynamics with two flavours of Wilson fermions on large lattices, arXiv:1210.6972.

[10] Y. Burnier and M. Laine, Towards flavour diffusion coefficient and electrical conductivity without ultraviolet contamination, Eur.Phys.J. C72 (2012) 1902, [arXiv:1201.1994].

[11] S. Gupta, The electrical conductivity and soft photon emissivity of the QCD plasma, Phys. Lett. $\mathbf{B 5 9 7}$ (2004) 57-62, [hep-lat/0301006].

[12] G. Aarts, C. Allton, J. Foley, S. Hands, and S. Kim, Spectral functions at small energies and the electrical conductivity in hot, quenched lattice QCD, Phys. Rev. Lett. 99 (2007) 022002, [hep-lat/0703008]. 